\title{
Nonlinear extensions of the Dirac equation and their implications in QED
}

\author{
D. C. Ionescu, J. Reinhardt, B. Müller, and W. Greiner \\ Institut für Theoretische Physik, J. W. Goethe-Universität, Robert-Mayer-Strasse 8-10, \\ D-6000 Frankfurt am Main, Federal Republic of Germany \\ G. Soff \\ Gesellschaft für Schwerionenforschung (GSI), Planckstrasse 1, Postfach 110 552, D-6100 Darmstadt, Federal Republic of Germany
}

(Received 23 December 1987)

\begin{abstract}
We investigate the influence of additional nonlinear terms in the Dirac Lagrangian on strongly bound electron states in heavy and superheavy atoms. Upper bounds for the coupling constants are deduced by comparison with precision spectroscopy data in QED. We demonstrate that nonlinear interactions may cause significant modifications of electron binding energies in superheavy quasiatomic systems which would not be visible in ordinary atomic-physics measurements.
\end{abstract}

In this paper we investigate the influence of additional contact interactions of Fermi-type in the Lagrangian of the classical Dirac field on electron binding energies in high- $Z$ collision systems. Let us assume the following relativistically invariant Lagrange density consisting of two parts:

$\mathcal{L}=\mathcal{L}_{D}+\mathcal{L}_{I}=\bar{\Psi}\left(i \gamma^{\mu} \partial_{\mu}-e \gamma^{\mu} A_{\mu}-m\right) \Psi+\lambda(\bar{\Psi} \Gamma \Psi)^{n}$,

where the first term $\mathcal{L}_{D}$ describes the interaction of the spinor field $\Psi$ with the electromagnetic field $A_{\mu}$ which is minimally coupled. The self-interaction term $\mathcal{L}_{I}$ is given by bilinear covariants of the Dirac theory. The coupling constant $\lambda$ has to be real in order to ensure a conserved total probability. Let us assume $\lambda>0$ such that the second term in (1) is attractive, and consider in the standard representation of the Dirac matrices ${ }^{1}$ the cases $\Gamma_{s}=I_{4 \times 4}$ (scalar coupling) and $\Gamma_{V}=\gamma^{\mu}$ (vector coupling). Such nonlinear interactions were studied within the last few years because the resulting nonlinear field equations possess solitary wave solutions of finite energy and momentum. This property has been utilized by several authors to generate models for extended elementary particles. Some of the extensive theoretical research work in this field is cited in Ref. 2. We also mention that in his attempts to construct a unified theory of elementary particles, Heisenberg proposed a nonlinear Dirac equation for quantized spinor fields. ${ }^{3}$

A second motivation of our investigations arose from the observed sharp line structures ${ }^{4}$ in the spectra of positrons emitted from superheavy collision systems. Similar line structures have already been predicted in connection with the decay of the neutral vacuum in QED. ${ }^{5}$ In various experiments performed at Gesellschaft für Schwerionenforschung (GSI), peaklike structures have been discovered at impact energies close to the Coulomb barrier, but they cannot be identified with the mechanism of spontaneous $e^{+}-e^{-}$pair creation. For a review of the experimental status see Ref. 4 .
A great variety of theoretical models has been proposed during the past years to explain these peaklike structures (many references of corresponding recent theoretical work are provided in Ref. 6). It also has been suggested $^{7}$ that a system containing several electronpositron pairs could explain the observed correlated emission of electrons and positrons in coincidence experiments. Such a polypositronium state could be highly localized and tightly bound if one assumes the existence of a short-range many-body interaction. Such a new nonlinear many-body force is required to be sufficiently weak to provide negligible corrections in the ordinary atomicphysics sector. For example, it could grow like $\lambda \rho^{n}$, where $\rho$ is the electronic density.

A further important contribution related to the new phenomenon of spontaneous positron formation in QED was the investigation of self-energy and vacuum polarization corrections ${ }^{8,9}$ and some new types of interactions between leptons in the electromagnetic field ${ }^{10}$ and their influence on electron binding energies in superheavy collision systems. However, calculations displayed no significant shift of the electron binding energies.

Since the Lagrangian (1) describes a nonrenormalizable theory for $n>\frac{4}{3}$, it must be regarded as an effective model in the low-energy sector of a more fundamental renormalizable interaction. We note that the Lagrange density (1) satisfies the relation

$$
\bar{\Psi}\left(\frac{\partial \mathcal{L}_{I}}{\partial \bar{\Psi}}\right)=\left(\frac{\partial \mathcal{L}_{I}}{\partial \Psi}\right) \Psi=n \mathcal{L}_{I} .
$$

Furthermore, if $\Psi(x)$ is a solution of the variational problem, one easily obtains the following functional relation between the total Lagrange density $\mathcal{L}$ and the selfinteraction part $\mathcal{L}_{I}$ from Eq. (1):

$$
\mathcal{L}[\Psi]=(1-n) \mathcal{L}_{I}[\Psi] .
$$

The total energy for stationary solutions, which is obtained from the $T^{00}$ component of the energy-momentum tensor $T^{\mu v}$, reads 


$$
\begin{aligned}
E[\Psi] & =\int d^{3} x\left(i \bar{\Psi} \gamma^{0} \partial_{0} \Psi-\mathcal{L}\right) \\
& =\omega \int d^{3} x \psi^{\dagger} \psi+(n-1) \int d^{3} x \mathcal{L}_{I},
\end{aligned}
$$

where we employed the relation (2) and the separation of the time dependence of the Dirac field $\Psi$ due to

$$
\Psi(t, \mathbf{x})=\psi(\mathbf{x}) e^{-i \omega t} .
$$

The frequency $\omega$ represents a parameter describing the time evolution of the spinor field $\Psi$. Note that in the linear theory $(\lambda=0)$ one has $E=\omega$.

The variational principle yields, from (1), the following field equation for the spinor field $\Psi(x)$ :

$\left[i \gamma^{\mu} \partial_{\mu}-e \gamma^{\mu} A_{\mu}-m+n \lambda(\bar{\Psi} \Gamma \Psi)^{n-1} \Gamma\right] \Psi(x)=0$.

We now consider the case of a spherically symmetric electrostatic potential of a homogeneously charged sphere, i.e., $A_{\mu}=\left(A_{0}(r), 0\right)$, and construct continuous normalizable stationary solutions of the nonlinear Dirac equation (6) which are simultaneously eigenstates of the total angular momentum, parity, and time evolution operators. For these solutions we make the usual spherical separation ansatz,

$$
\Psi_{j m}^{l}(t, \mathbf{x})=\exp \left(-i \omega_{j m}^{l} t\right)\left(\begin{array}{c}
i g_{j l}(r) \Omega_{j m}^{l}(\vartheta, \varphi) \\
f_{j l}(r) \widehat{\sigma}_{r} \Omega_{j m}^{l}(\vartheta, \varphi)
\end{array}\right]
$$

where $g_{j l}$ and $f_{j l}$ are radial functions and $\Omega_{j m}^{l}(\vartheta, \varphi)$ are two-component spherical spinors defined as in Refs. 1 . They contain the angular dependence of the spinor field $\Psi$. $\hat{\sigma}_{r}$ denotes a scalar operator which ensures that the upper and lower components in (7) have opposite parity. Inserting the ansatz (7) into the field equation (6) yields stationary coupled equations for scalar $(S)$ and vector $(V)$ coupling, respectively, which possess spherical symmetric solutions only for states with $j=\frac{1}{2}$ and $l=0,1$, i.e., $s_{1 / 2}$ and $p_{1 / 2}$ states. Here the resulting eigenvalue equations are separable. By inspection of the self-interaction term in Eq. (6) we conclude that for vector coupling $\left(\Gamma_{V}=\gamma^{\mu}\right)$, only the timelike component $(\mu=0)$ leads to spherically symmetric solutions. With the substitutions

$$
\begin{aligned}
& S_{n}(g, f)=n\left[\frac{g^{2}-f^{2}}{4 \pi}\right)^{n-1}, \\
& V_{n}(g, f)=n\left(\frac{g^{2}+f^{2}}{4 \pi}\right)^{n-1},
\end{aligned}
$$

which represent the scalar $(S)$ and vector $(V)$ selfinteractions, respectively, Eq. (6) in spherical coordinates yields the following radial equations of motion for the lowest-energy state with $j=\frac{1}{2}, \quad m= \pm \frac{1}{2}$, and $l=0$ $(\hbar=c=1$ ). For scalar coupling,

$$
\frac{d}{d r}\left(\begin{array}{l}
g(r) \\
f(r)
\end{array}\right)=\left(\begin{array}{cc}
0 & \omega-e A_{0}(r)+m-\lambda_{s} S_{n} \\
-\omega+e A_{0}(r)+m-\lambda_{s} S_{n} & -2 / r
\end{array}\right)\left(\begin{array}{l}
g(r) \\
f(r)
\end{array}\right) .
$$

For vector coupling,

$$
\frac{d}{d r}\left[\begin{array}{l}
g(r) \\
f(r)
\end{array}\right]=\left(\begin{array}{cc}
0 & \omega-e A_{0}(r)+\lambda_{v} V_{n+m} \\
-\omega+e A_{0}(r)-\lambda_{v} V_{n+m} & -2 / r
\end{array}\right]\left[\begin{array}{l}
g(r) \\
f(r)
\end{array}\right] .
$$

In addition to these equations we have the normalization condition of the spinor field $\Psi$, i.e.,

$$
\int d^{3} x \Psi^{\dagger} \Psi=\int_{0}^{\infty} d r r^{2}\left[g^{2}(r)+f^{2}(r)\right]=1 .
$$

Inserting this normalization condition into Eq. (4) we obtain the following relation between the total energy $E$ and the eigenvalue parameter $\omega$ from the systems (10) and (11):

$$
\begin{aligned}
E & =\omega+(n-1) \int d^{3} x \mathcal{L}_{I} \\
& =\omega+\lambda \frac{n-1}{(4 \pi)^{n-1}} \int_{0}^{\infty} d r r^{2}\left[g^{2}(r) \pm f^{2}(r)\right]^{n} .
\end{aligned}
$$

In the second term of Eq. (13) the plus and minus signs correspond to vector $(V)$ and scalar $(S)$ coupling, respectively. We now solve the systems (10) and (11) for the cases $n=2$ and 3 for scalar coupling and $n=2$ for vector coupling. Note that vector-type self-interaction terms with odd $n$ would lead to theories which are not Lorentz invariant. As it is not possible to find analytical solu- tions $^{11}$ a numerical analysis has to be performed. We have solved the eigenvalue problem of the system of radial equations (10) and (11) with the integral constraint (12) using the general-purpose computer code COLSYS written by Ascher, Christiansen, and Russel. ${ }^{12}$ The eigenvalue problem has been converted into a system of ordinary differential equations with boundary conditions expressed at the two end points by defining a new function $N(r)$,

$$
N(r)=\int_{0}^{r} d r_{1} r_{1}^{2}\left[g^{2}\left(r_{1}\right)+f^{2}\left(r_{1}\right)\right]
$$

which leads to the additional differential equation

$$
\frac{d}{d r} N(r)=r^{2}\left[g^{2}(r)+f^{2}(r)\right]
$$

with the boundary conditions at the end points

$$
N(0)=0, \quad N(\infty)=1 .
$$

For numerical purposes the unknown frequency eigenval- 
ue $\omega$ is regarded as a further independent function, satisfying

$$
\frac{d \omega}{d r}=0
$$

In this way we have transformed the eigenvalue problem (10), (11), and (12) into a boundary-value problem which is defined by the four differential equations (10), (11), (15), and (17), with the corresponding boundary conditions (16), together with $g(0)=0, f(\infty)=0$.

For further investigations it is now crucial to note that the values of the coupling constants $\lambda_{s}$ and $\lambda_{v}$ are stringently limited by the precise data from atomic spectroscopy. Therefore we have investigated the consequences of the considered self-interaction terms in the following three cases.

(i) Lamb shift in hydrogen, $\left|E_{\text {expt }}-E_{\text {theor }}\right|<0.03$ MHz.

(ii) $K \alpha$ transition energy in ${ }^{100} \mathrm{Fm}$, $\left|E_{\text {expt }}-E_{\text {theor }}\right|<10 \mathrm{eV}$.

(iii) $K \alpha$ transition energy in hydrogenlike ${ }^{36} \mathrm{Kr}$, $\left|E_{\text {expt }}-E_{\text {theor }}\right|<0.5 \mathrm{eV}$.

The energy differences $\left|E_{\text {expt }}-E_{\text {theor }}\right|$ represent the present agreement between experimental values and theoretical predictions. ${ }^{13}$ From these transition energies we deduce upper limits for the coupling constants $\lambda$. The additional terms in Eq. (1) have no significant consequences in the ordinary atomic physics up to $Z=100$ if the coupling constants do not exceed the maximal allowed values $\lambda_{\max }$ indicated in Table $I$. Note that in this calculation only the self-interaction terms were considered.

With the determined upper bounds for the coupling constants, we solve the systems of equations (10), (11), (15), and (17) for $K$-shell electrons in superheavy atoms. The last column in Table $I$ indicates the influence of the additional nonlinear terms on the energy of a $K$-shell electron in the superheavy quasiatom with $Z=155$ for $\lambda=\lambda_{\max }$. In the case of scalar coupling with $n=3$ one obtains a large shift of the $K$-shell energy by about $200 \mathrm{keV}$. Similar calculations have been presented in Refs. 10 and 15 for $n=2$. These authors also verified that first-order perturbation theory is no longer applicable in the case of such strong nonlinear interactions.

TABLE I. Upper limits for the coupling constants consistent with experimental transition energies in atoms for several nonlinear interactions. We note that the $K \alpha$ transition energy in ${ }^{100} \mathrm{Fm}$ provides the most stringent limit $\lambda_{\max }$. The last column indicates the $1 s_{1 / 2}$-level shift in a $Z=155$ quasiatom for $\lambda=\lambda_{\max }$. The coupling constants have in natural units the dimension of a power of the length, i.e., $[L]^{3 n-4}$.

\begin{tabular}{ccc}
\hline \hline Coupling & $\lambda_{\max }\left([L]^{3 n-4}\right)$ & $\Delta E(\mathrm{keV})$ \\
\hline$S: \lambda_{s}(\bar{\Psi} \Psi)^{2}$ & $4.7 \times 10^{-4}$ & 3 \\
$S: \lambda_{s}(\bar{\Psi} \Psi)^{3}$ & $2.3 \times 10^{-3}$ & 200 \\
$V: \lambda_{v}\left(\bar{\Psi} \gamma^{0} \Psi\right)^{2}$ & $2.2 \times 10^{-4}$ & 7 \\
\hline \hline
\end{tabular}

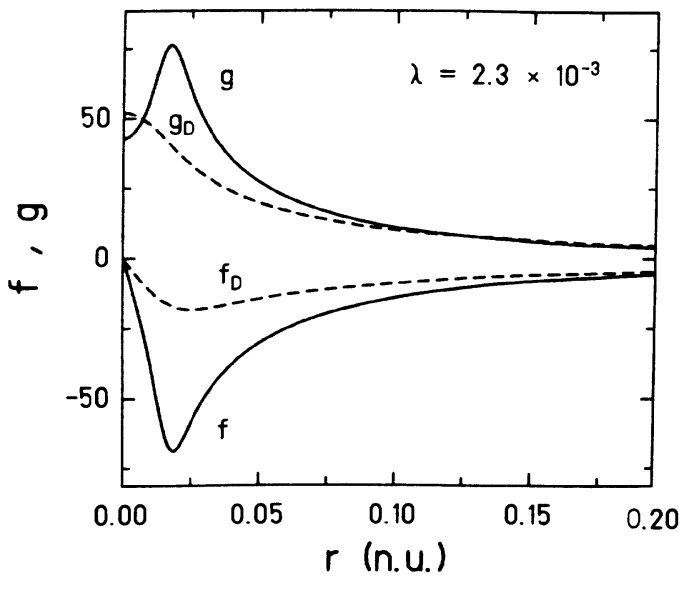

FIG. 1. Radial functions for the $1 s_{1 / 2}$ state of a $Z=155$ quasiatom with cubic nonlinearity. The solid curves correspond to the nonlinear Dirac equation taking the maximal allowed value for $\lambda$. The dashed lines indicate the radial functions of the linear theory.

Typical shapes for the radial functions $g$ and $f$ are depicted in Fig. 1. The displayed radial functions demonstrate that the solutions are highly localized due to the fact that the self-interaction terms can be regarded as additional attractive potentials. This effect can also be recognized in the density plot illustrated in Fig. 2. We note that the large component $|g|$ decreases, while the small component $|f|$ increases, with growing values of the coupling constant due to the nonlinearities, such that they become similar in shape and magnitude. For scalar couplings with $n=3$ the influence of the nonlinear terms decreases if the coupling constant exceeds a certain value, because of the combination $\left(g^{2}-f^{2}\right)^{2}$ in the radial equations (10). Hence we expect a kind of saturation in the

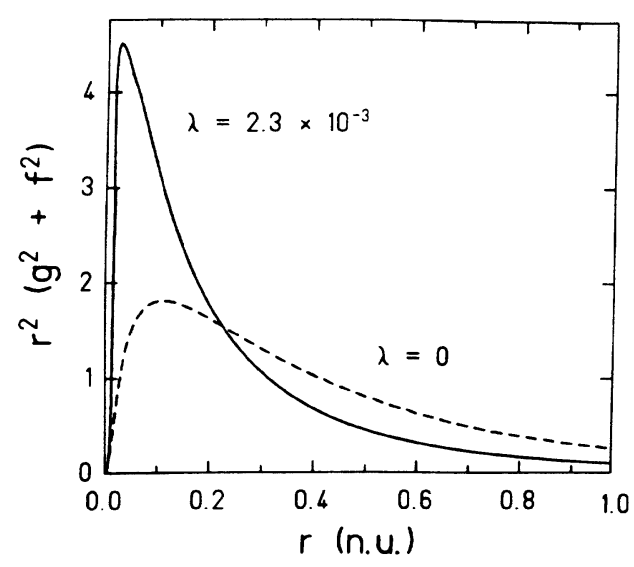

FIG. 2. Scalar densities in a quasiatom with $Z=155$ for the lowest-energy state. The dashed line corresponds to the linear theory $(\lambda=0)$, while the solid line belongs to the nonlinear model with cubic nonlinearity for $\lambda=\lambda_{\max }$. $\lambda$ is given natural units (n.u.) $(\hbar=m=c=1)$. 


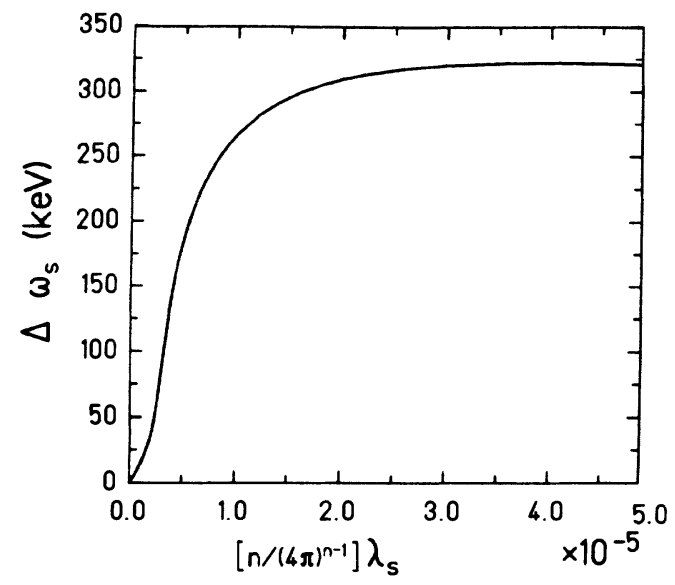

FIG. 3. The frequency eigenvalue shift $\Delta \omega_{s}=\omega(0)-\omega(\lambda)$ in dependence on the coupling constant $\left[n /(4 \pi)^{n-1}\right] \lambda_{s}$ in a $Z=156$ quasiatom assuming a $(\bar{\Psi} \Psi)^{3}$ coupling.

variation of the frequency eigenvalue $\omega$ with $\lambda$, which is presented in Fig. 3.

Figure 4 illustrates the main result of our investigation, viz., the influence of the scalar self-interaction $(\bar{\Psi} \Psi)^{3}$ on the total energy of a $K$-shell electron in superheavy quasiatoms up to $Z=190$, taking the maximally allowed value for the coupling constant $\lambda_{s}$ from spectroscopic precision experiments (see Table I). The total energies are calculated from Eq. (13).

The solid line represents the energy of the $1 s_{1 / 2}$ state predicted by the Dirac theory, assuming a Coulomb potential of an extended nucleus where the nuclear radius $R_{\text {nuc }}$ is assumed to be $R_{\text {nuc }}=1.2 A^{1 / 3} \mathrm{fm}$. The $1 s_{1 / 2}$ state joins the negative-energy continuum at the critical charge $Z_{\text {crit }} \simeq 170 .^{5}$ In the supercritical region the $1 s$-resonance

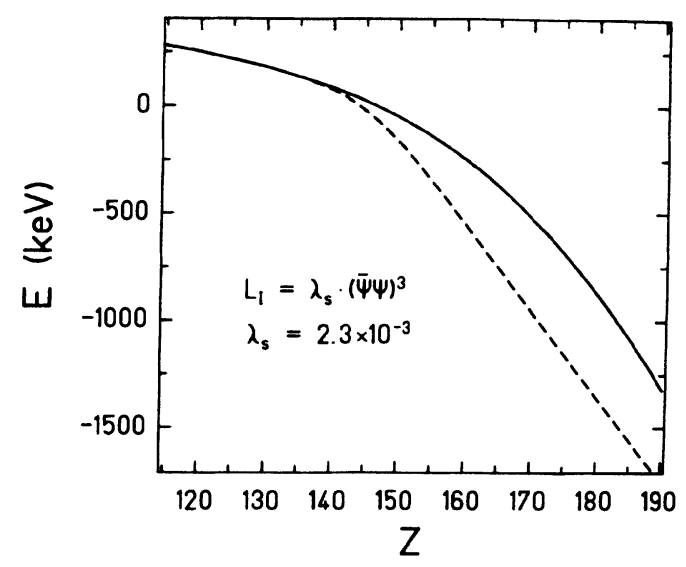

FIG. 4. Total energies of $1 s_{1 / 2}$ electrons in atoms up to $Z=190$. The solid curve represents the predictions of the Dirac theory $(\lambda=0)$, while the dashed curve indicates the modified $K$ shell energies due to cubic self-interaction terms $\lambda_{s}(\bar{\Psi} \Psi)^{3}$, taking the maximal allowed values for $\lambda_{s}$.
TABLE II. Upper bounds for the coupling constants consistent with precision experiments assuming a $\lambda_{1} \mathbf{E}^{2}(\bar{\Psi} \Gamma \Psi)^{n}$ interaction.

\begin{tabular}{cc}
\hline \hline Coupling & $\lambda_{1}\left([L]^{3 n-4}\right)$ \\
\hline$S: g_{s}(\bar{\Psi} \Psi)^{2}$ & $2.3 \times 10^{-10}$ \\
$S: g_{s}(\bar{\Psi} \Psi)^{3}$ & $1.3 \times 10^{-10}$ \\
$V: g_{v}\left(\bar{\Psi} \gamma^{0} \Psi\right)^{2}$ & $1.3 \times 10^{-10}$ \\
\hline \hline
\end{tabular}

energies have been determined by an approximate cutoff procedure. ${ }^{14}$ The results of our calculations for the nonlinear Dirac equation are illustrated by the dashed line. We observe that the influence of the nonlinear interactions is negligible for $Z<135$. However, if the charge is continuously increased, the modifications of the $K$-shell energy become quite significant. In this region the energy shift amounts to about $200 \mathrm{keV}$. This indicates that high- $Z$ systems are quite sensitive to such types of interactions.

Finally, we investigated three other more exotic interactions given by the interaction Lagrangians

$$
\begin{aligned}
& \mathcal{L}_{1}=\lambda_{1} \mathbf{E}^{2}(\bar{\Psi} \Gamma \Psi)^{n}, \\
& \mathcal{L}_{2}=\lambda_{2}(\bar{\Psi} \Gamma \Psi)^{n} \exp (\bar{\Psi} \Gamma \Psi)^{n}, \\
& \mathcal{L}_{3}=m \bar{\Psi} \Psi \exp \left(-\frac{\lambda_{3}}{m}(\bar{\Psi} \Psi)^{2}\right),
\end{aligned}
$$

where $\mathbf{E}$ denotes the electric field strength of the positively charged nucleus of the superheavy atom. In Eq. (20), $m$ represents the rest mass of the electron. We consider again the two cases $\Gamma_{s}=I_{4 \times 4}$ (scalar coupling) and $\Gamma_{V}=\gamma^{0}$, respectively, and calculate upper bounds for the coupling constants $\lambda_{1}, \lambda_{2}$, and $\lambda_{3}$ from the requirement that the discrepancy between theoretical and experimental values of the $K \alpha$ transition energy has to be less than $10 \mathrm{eV}$ in the element ${ }^{100} \mathrm{Fm}$. The results of this calculation are presented in Tables II and III for the additional interactions (18) and (19), respectively. Note that in case (20) one actually has an effective density-dependent mass, i.e.,

$$
m_{\text {eff }}=m\left[\exp \left[-\frac{\lambda_{3}}{m}(\bar{\Psi} \Psi)^{2}\right]\right] .
$$

From this, the Taylor expansion of $m_{\text {eff }}$ reads

TABLE III. Upper bounds for the coupling constants consistent with precision experiments assuming a $\lambda_{2}$ $(\bar{\Psi} \Gamma \Psi)^{n} \exp (\bar{\Psi} \Gamma \Psi)$ interaction.

\begin{tabular}{lll}
\hline \hline Coupling & $n$ & $\lambda_{2}\left([L]^{3 n-4}\right)$ \\
\hline$S: \hat{\Gamma}_{S}=I$ & 2 & $3.7 \times 10^{-4}$ \\
$S: \hat{\Gamma}_{S}=I$ & 3 & $1.3 \times 10^{-3}$ \\
$S: \hat{\Gamma}_{S}=I$ & 2 & $2.6 \times 10^{-4}$ \\
$S: \hat{\Gamma}_{S}=I$ & 3 & $5.5 \times 10^{-4}$ \\
$V: \hat{\Gamma}_{V}=\gamma^{0}$ & 2 & $1.6 \times 10^{-4}$ \\
$V: \hat{\Gamma}_{V}=\gamma^{0}$ & 2 & $1 \times 10^{-4}$ \\
\hline \hline
\end{tabular}




$$
m_{\mathrm{eff}}=m-\lambda_{3}(\bar{\Psi} \Psi)^{2}+\frac{1}{2} \frac{\lambda_{3}^{2}}{m}(\bar{\Psi} \Psi)^{4} \pm \cdots
$$

This interaction represents, in fact, a superposition of scalar self-interactions with different even powers. We obtained a maximally allowed value $\lambda_{3} \simeq 2 \times 10^{-3}$ n.u. By comparison with the maximal $\lambda_{s}$ value from Table I one can conclude that these two bounds are nearly equal. This result verifies that higher-order terms in the expansion (22) are of no importance. Only the dominant term $\lambda_{3}(\bar{\Psi} \Psi)^{2}$ plays a major role.

Finally, we summarize the main results of our investigation. We have studied the influence of additional nonlinear terms in the Dirac Lagrangian on electron binding energies of very heavy atoms with combined nuclear charge up to $Z=190$, which can be transiently formed in heavy-ion collisions. From QED precision experiments we determined upper bounds for the corresponding coupling constants. Taking the maximally allowed values for the coupling constants, a simple nonlinear interaction $\mathcal{L}_{I}=\lambda_{s}(\bar{\Psi} \Psi)^{3}$ was found to provide a rather strong modification of binding energies of $K$-shell electrons in superheavy quasiatoms with $Z>150$. Such an interaction causes, for all known atoms in the Periodic Table, a negligible modification of the $K$-shell energy. Theoretical investigations ${ }^{16}$ have shown that the excitation probability $P_{1 s \sigma}(b)$ of the molecular $1 s \sigma$ orbital in encounters with impact parameter $b$ can be represented with good accuracy by the expression

$$
P(b)=D(Z) \exp \left[-E_{b}\left(R_{0}\right) / \Gamma(b)\right],
$$

where $E_{b}\left(R_{0}\right)$ is the $1 s \sigma$ binding energy and $R_{0}$ is the distance of closest approach of two very heavy ions $Z_{1}$ and $Z_{2}$. Details on the strength function $D(Z)$ and the falloff constant $\Gamma(b)$ are given in Ref. 16. This law has been proposed as a tool to determine the binding energies of the $1 s \sigma$ orbitals by the measurement ${ }^{17}$ of the excitation probability $P_{1 s \sigma}(b)$ as a function of the impact parameter $b$ for several collision systems with $128<Z_{1}+Z_{2}<179$. In consequence, precision ionization measurements in superheavy collision systems could further restrict the upper bounds of such additional nonlinear interactions.
${ }^{1}$ J. D. Bjorken and S. D. Drell, Relativistic Quantum Mechanics (McGraw-Hill, New York, 1964); W. Greiner, Vol. 6 of Theoretische Physik. Relativistische Quantenmechanik: Wellengleichungen, 2nd ed. (Deutsch, Frankfurt am Main, 1987).

${ }^{2}$ A. F. Rañada, in Quantum Theory Groups-Fields and Particles, edited by A. O. Barut (Reidel, Dordrecht, 1983), p. 241.

${ }^{3}$ W. Heisenberg, Rev. Mod. Phys. 29, 269 (1957).

${ }^{4}$ T. Cowan, H. Backe, K. Bethge, H. Bokemeyer, H. Folger, J. S. Greenberg, K. Sakaguchi, D. Schwalm, J. Schweppe, K. E. Stiebing, and P. Vincent, Phys. Rev. Lett. 56, 444 (1986); W. Koenig, F. Bosch, P. Kienle, C. Kozhuharov, H. Tsertos, E. Berdermann, S. Huchler, and W. Wagner, Z. Phys. A 328, 129 (1987).

${ }^{5}$ W. Greiner, B. Müller, and J. Rafelski, Quantum Electrodynamics of Strong Fields (Springer, Berlin, 1986).

${ }^{6} \mathrm{~J}$. Reinhardt, K. Geiger, M. Grabiak, D. C. Ionescu, A. Scherdin, S. Schramm, B. Müller, W. Greiner, and G. Soff, in Frontiers of Heavy-Ion Physics, edited by N. Cindro, W. Greiner, and R. Čaplar (World Scientific, Singapore, 1987), p. 409.

${ }^{7}$ B. Müller, J. Reinhardt, W. Greiner, and Schäfer, J. Phys. G 12, L109 (1986).

${ }^{8}$ G. Soff, P. Schlüter, B. Müller, and W. Greiner, Phys. Rev. Lett. 48, 1465 (1982).

${ }^{9}$ M. Gyulassy, Nucl. Phys. A244, 497 (1975).
${ }^{10}$ G. Soff, B. Müller, J. Rafelski, and W. Greiner, Z. Naturforsch. 28a, 1389 (1973).

${ }^{11}$ W.-H. Steeb, W. Oevel, and W. Strampp, J. Math. Phys. 25, 2331 (1984).

${ }^{12}$ U. Ascher, J. Christiansen, and R. D. Russel, Math. Comput. 33, 659 (1979).

${ }^{13}$ M. H. Chen, B. Crasemann, M. Aoyagi, K.-N. Huang, and H. Mark, At. Data and Nucl. Data Tables 26, 561 (1981); S. R. Lundeen and S. N. Pipkin, Phys. Rev. Lett. 46, 232 (1981); M. Tavernier, J. P. Briand, P. Indelicato, D. Liesen, and P. Richard, J. Phys. B 18, L327 (1985).

${ }^{14}$ J. Reinhardt, B. Müller, and W. Greiner, Phys. Rev. A 24, 103 (1981).

${ }^{15}$ G. Soff, U. Müller, S. Schramm, T. de Reus, G. Mehler, J. Reinhardt, B. Müller, and W. Greiner, in Atoms in Unusual Situations, Vol. 143 of NATO Advanced Study Institute, Series B: Physics, edited by J. P. Briand (Plenum, New York, 1986), p. 343.

${ }^{16}$ G. Soff, B. Müller, and W. Greiner, Phys. Rev. Lett. 40, 540 (1978).

${ }^{17}$ D. Liesen, P. Armbruster, F. Bosch, S. Hagmann, P. H. Mokler, H. J. Wollersheim, H. Schmidt-Böcking, R. Schuch, and J. B. Wilhelmy, Phys. Rev. Lett. 44, 983 (1980). 